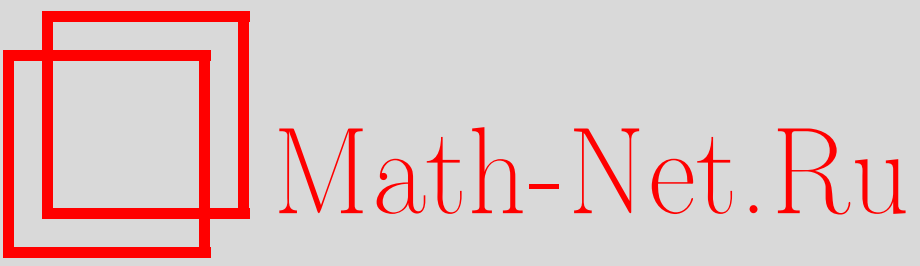

С. Гравель, Суперинтегрируемые системы с интегралами третьего порядка в классической и квантовой механике, ТМФ, 2003, том 137, номер 1, 97-107

DOI: https://doi.org/10.4213/tmf248

Использование Общероссийского математического портала Math-Net.Ru подразумевает, что вы прочитали и согласны с пользовательским соглашением

http://www . mathnet.ru/rus/agreement

Параметры загрузки:

IP : 34.239 .49 .27

26 апреля 2023 г., 16:53:56 


\author{
ТЕОРЕТИЧЕСКАЯ \\ И МАТЕМАТИЧЕСКАЯ \\ ФИЗИКА \\ Том 137, № 1 \\ октябрь, 2003
}

(C) 2003 г.

\author{
С. Гравель*
}

\title{
СУПЕРИНТЕГРИРУЕМЫЕ СИСТЕМЫ С ИНТЕГРАЛАМИ ТРЕТЬЕГО ПОРЯДКА В КЛАССИЧЕСКОЙ И КВАНТОВОЙ МЕХАНИКЕ
}

В пространстве $E(2)$ дан обзор систем, допускающих разделение переменных в декартовых координатах и имеющих интеграл движения третьего порядка как в квантовой, так и в классической механике. Отличия и сходные черты этих двух случаев проиллюстрированы многочисленными примерами. Многие из построенных суперинтегрируемых систем оказываются новыми, и можно увидеть связь между суперинтегрируемыми потенциалами и трансцендентами Пенлеве.

Ключевые слова: интегралы движения, суперинтегрируемость, разделение переменных.

\section{1. ВВЕ ДЕНИЕ}

В классической механике $n$-мерная гамильтонова система называется интегрируемой по Лиувиллю, если она допускает $n$ функционально независимых интегралов движения, находящихся в инволюции (включая гамильтониан), т.е.

$$
\begin{aligned}
\left\{H, X_{i}\right\} & =0, \\
\left\{X_{i}, X_{j}\right\} & =0
\end{aligned}
$$

для всех $i, j$. Как гамильтониан $H=H\left(x_{1}, \ldots, x_{n}, p_{1}, \ldots, p_{n}\right)$, так и интегралы движения $X_{i}=X_{i}\left(x_{1}, \ldots, x_{n}, p_{1}, \ldots, p_{n}\right)$ должны быть хорошо определенными функциями на фазовом пространстве [1]. Система называется суперинтегрируемой, если она допускает более чем $n$ функционально независимых интегралов движения, из которых $n$ находятся в инволюции. Система называется максимально суперинтегрируемой, если она допускает $2 n-1$ интегралов движения.

В квантовой механике гамильтонова система называется интегрируемой, если существует набор $\left\{X_{i}\right\}$, состоящий из $n$ хорошо определенных алгебраически независимых операторов (включая гамильтониан), которые попарно коммутируют. Система будет

${ }^{*}$ CRM, Université de Montréal, C.P.-6128, Succursale Centre-Ville, Montreal, Quebec H3C 3J7 Canada. E-mail: graves@magellan.umontreal.ca 
суперинтегрируемой, если у нее к тому же есть независимые операторы $\left\{Y_{j}\right\}$, коммутирующие с гамильтонианом. Операторы $Y_{j}$ не обязаны коммутировать ни между собой, ни с операторами $X_{i}$.

Такое определение квантовой интегрируемости предполагает, что само понятие независимости квантовых операторов хорошо определено, хотя в этой области не сушествует никакого идеально удовлетворительного определения независимости. Тем не менее по аналогии с классическим случаем можно определить, какие из интегралов движения должны рассматриваться как независимые, имея в виду то, что при формулировании общих утверждений может потребоваться и точное определение независимости.

Проблема понимания и классификации интегрируемых и суперинтегрируемых гамильтоновых систем в классической механике исследовалась в течение долгого времени. Поиск квантовых интегрируемых [2]-[4] и суперинтегрируемых [5], [6] систем представляет собой более новую отрасль знаний. В большинстве работ рассматривались классические системы с интегралами второго порядка в двумерном или $n$-мерном плоском пространстве [7] или в пространствах с постоянной [8] или переменной [9] кривизной. При условии, что гамильтониан не содержит зависимости от скоростей, никаких отличий между свойствами квантовой и классической интегрируемости в этих случаях обнаружено не было. Более того, была выявлена глубокая связь между такой “квадратичной суперинтегрируемостью", свойством разделимости переменных в уравнениях Шредингера и Гамильтона-Якоби, точной интегрируемостью [10] и обобщенными симметриями [5].

В немногочисленных работах также исследовались интегралы третьего и старших порядков в классической механике [11]-[13], и только в самое последнее время внимание исследователей привлекли квантовые интегралы движения третьего порядка, для которых было обнаружено, что эквивалентность между квантовым и классическим свойствами интегрируемости нарушается при рассмотрении интегралов движения старших порядков [2], [3], [14], [15]. Известные случаи суперинтегрируемых потенциалов, допускаюших сушествование интегралов движения старших порядков, все еше весьма редки как в квантовом, так и в классическом случаях.

Систематический поиск суперинтегрируемых систем с интегралами старших порядков был начат в работе [15]. Были найдены все потенциалы вида

$$
H=\frac{1}{2 m}\left(p_{1}^{2}+p_{2}^{2}\right)+V(x, y)
$$

которые допускают сушествование интегралов первого и третьего порядков; эти потенциалы обсуждаются в разделе 3 .

Логическим продолжением данной работы является поиск систем с интегралами второго и третьего порядков. Этот класс систем должен быть гораздо более общим, поскольку сушествование интеграла движения второго порядка оказывается гораздо менее ограничиваюшим свойством по сравнению с условием сушествования интеграла движения первого порядка. Как в классической, так и в квантовой механике условие существования интеграла движения второго порядка приводит к тому, что гамильтониан 
допускает разделение переменных в декартовых, полярных, параболических или эллиптических координатах. В разделе 4 приводятся предварительные результаты для потенциалов, допускаюших разделение переменных в декартовых координатах:

$$
H=\frac{1}{2 m}\left(p_{1}^{2}+p_{2}^{2}\right)+V_{1}(x)+V_{2}(y) .
$$

Полное описание таких интегралов находится в состоянии разработки.

\section{2. СУЩЕСТВОВАНИЕ ИНТЕГРАЛОВ ТРЕТЬЕГО ПОРЯДКА}

В квантовой и классической механике оператор третьего порядка обшего вида, коммутируюший с гамильтонианом, должен иметь вид

$$
X=\sum_{i+j=0}^{3} P_{i j}(x, y) p_{1}^{i} p_{2}^{j} .
$$

Как в классической, так и в квантовой механике с помощью прямых вычислений можно показать, что члены в лидирующих порядках (здесь это члены порядка два и три) должны лежать в обертываюшей алгебре группы $E(2)$. Более того, можно показать, что в случае классической механики члены с четными и нечетными степенями импульса не могут одновременно появляться ни в каком из определяюших уравнений, получаемых при наложении условия $\dot{X}=0$. Поэтому члены четного и нечетного порядка по степеням импульса должны входить в различные интегралы движения (которые, возможно, тривиальны). Так как в данной работе ишутся интегралы третьего порядка, можно положить члены четных порядков по степеням импульса равными нулю и искать ответ в виде

$$
\begin{aligned}
& X=\sum_{\substack{i, j, k \\
i+j+k=3}} A_{i j k} L_{3}^{i} p_{1}^{j} p_{2}^{k}+g_{1}(x, y) p_{1}+g_{2}(x, y) p_{2}, \\
& L_{3}=x p_{2}-y p_{1} .
\end{aligned}
$$

Как ожидается, такое же свойство должно проявляться и в квантовой механике, но в этом вопросе надо быть более осторожными. Во-первых, можно использовать антикоммутаторы для того, чтобы перестроить члены и привести интеграл к симметричному виду:

$$
\begin{aligned}
X= & \sum_{\substack{i, j, k \\
i+j+k=3}} A_{i j k}\left\{L_{3}^{i}, p_{1}^{j} p_{2}^{k}\right\}+\sum_{\substack{i, j, k \\
i+j+k=2}} B_{i j k}\left\{L_{3}^{i}, p_{1}^{j} p_{2}^{k}\right\}+ \\
& +\left\{g_{1}(x, y), p_{1}\right\}+\left\{g_{2}(x, y), p_{2}\right\}+h(x, y) .
\end{aligned}
$$

Далее, можно использовать самосопряженность гамильтониана, чтобы показать, что части, четные и нечетные по степеням импульса, должны коммутировать с гамильтонианом по отдельности. Поэтому оператор третьего порядка может быть представлен в 
виде

$$
X=\sum_{\substack{i, j, k \\ i+j+k=3}} A_{i j k}\left\{L_{3}^{i}, p_{1}^{j} p_{2}^{k}\right\}+\left\{g_{1}(x, y), p_{1}\right\}+\left\{g_{2}(x, y), p_{2}\right\}
$$

где $A_{i j k}$ - произвольные вешественные константы, а $g_{i}$ - произвольные вешественные функции. Использование антикоммутатора вкупе со свойством самосопряженности гамильтониана позволяет не только устранить члены четных степеней по $p_{i}$ в квантовой механике, но и обеспечить самосопряженность исследуемого оператора, что дает возможность четко проследить связь между квантовым и классическим случаями.

Наконец, отметим, что при подходящем изменении обозначений, а именно

$$
\begin{aligned}
V(x, y) & =\frac{\hbar^{2}}{2 m} \tilde{V}(x, y), \\
g_{1}(x, y) & =\hbar^{2} \tilde{g_{1}}(x, y), \\
g_{2}(x, y) & =\hbar^{2} \tilde{g_{2}}(x, y),
\end{aligned}
$$

условие $[H, X]=0$ в квантовой механике становится независимым от параметров $\hbar$ и $m$. Поэтому во всех уравнениях можно положить $\hbar=m=1$ и найти все решения для $\widetilde{V}, \tilde{g}_{1}$ и $\tilde{g}_{2}$, которые будут независимы от $\hbar$ и $m$. Отсюда следует, что все квантовые интегрируемые системы могут быть положены пропорциональными $\hbar^{2} / m$. Тем не менее отсюда вовсе не следует, что все квантовые интегрируемые системы исчезают в классическом пределе, поскольку в решение могут входить произвольные параметры, выбранные в таком виде, чтобы эффективный потенциал $\widetilde{V}$ расходился в пределе $\hbar \rightarrow 0$.

Используя это свойство, можно положить $m=1$, но не следует налагать условие $\hbar=1$, поскольку задача состоит в построении классического предела.

Если теперь потребовать, чтобы классический гамильтониан $\left(p_{1}^{2}+p_{2}^{2}\right) / 2+V(x, y)$ коммутировал по Пуассону с интегралом $X$, то получатся следующие условия:

$$
\begin{aligned}
0 & =g_{1} V_{x}+g_{2} V_{y}, \\
\left(g_{1}\right)_{x} & =3 f_{1}(y) V_{x}+f_{2}(x, y) V_{y} \equiv h_{1}, \\
\left(g_{2}\right)_{y} & =f_{3}(x, y) V_{x}+3 f_{4}(x) V_{y} \equiv h_{2}, \\
\left(g_{1}\right)_{y}+\left(g_{2}\right)_{x} & =2\left(f_{2}(x, y) V_{x}+f_{3}(x, y) V_{y}\right) \equiv h_{3},
\end{aligned}
$$

где

$$
\begin{aligned}
f_{1}(y) & =-A_{300} y^{3}+A_{210} y^{2}-A_{120} y+A_{030}, \\
f_{2}(x, y) & =3 A_{300} x y^{2}-2 A_{210} x y+A_{201} y^{2}+A_{120} x-A_{111} y+A_{021}, \\
f_{3}(x, y) & =-3 A_{300} x^{2} y+A_{210} x^{2}-2 A_{201} x y+A_{111} x-A_{102} y+A_{012}, \\
f_{4}(x) & =A_{300} x^{3}+A_{201} x^{2}+A_{102} x+A_{003} .
\end{aligned}
$$


$\mathrm{B}$ тех же обозначениях условие $[H, X]=0$ в квантовой механике приводит к системе уравнений

$$
\begin{gathered}
0=g_{1} V_{x}+g_{2} V_{y}-\frac{\hbar^{2}}{4}\left(f_{1} V_{x x x}+f_{2} V_{x x y}+f_{3} V_{x y y}+f_{4} V_{y y y}+\right. \\
\left.+8 A_{300}\left(x V_{y}-y V_{x}\right)+2\left(A_{210} V_{x}+A_{201} V_{y}\right)\right) \\
\left(g_{1}\right)_{x}=3 f_{1}(y) V_{x}+f_{2}(x, y) V_{y} \equiv h_{1} \\
\left(g_{2}\right)_{y}=f_{3}(x, y) V_{x}+3 f_{4}(x) V_{y} \equiv h_{2}, \\
\left(g_{1}\right)_{y}+\left(g_{2}\right)_{x}=2\left(f_{2}(x, y) V_{x}+f_{3}(x, y) V_{y}\right) \equiv h_{3} .
\end{gathered}
$$

Уравнения классической механики получаются из этой системы простой подстановкой $\hbar=0$. Следовательно, можно ожидать, что решения будут хорошо вести себя в классическом пределе. Известно также, что обшее решение системы уравнений (2.7)(2.10) будет содержать в себе все решения уравнений (2.3)-(2.6), т.е. классическое решение оказывается частным случаем квантового. Отметим, что первое из уравнений $(2.7)$ нелинейно, чего не наблюдалось в случае интегралов низших порядков. Из этого уравнения должны следовать нелинейные условия согласования для потенциала. Наконец, из этих систем уравнений следует, что классические интегрируемые потенциалы могут быть умножены на произвольную константу (оставаясь интегрируемыми), в то время как допускающие это свойство квантовые потенциалы должны быть одновременно интегрируемыми потенщиалами классической задачи.

Три последних уравнения, совпадаюшие в квантовом и классическом случаях, задают линейное условие согласованности для потенциала $V$, имеющее вид

$$
\begin{aligned}
0= & -f_{3} V_{x x x}+\left(2 f_{2}-3 f_{4}\right) V_{x x y}+\left(-3 f_{1}+2 f_{3}\right) V_{x y y}-f_{2} V_{y y y}+ \\
& +2\left(f_{2 y}-f_{3 x}\right) V_{x x}+2\left(-3 f_{1 y}+f_{2 x}+f_{3 y}-3 f_{4 x}\right) V_{x y}+2\left(-f_{2 y}+f_{3 x}\right) V_{y y}+ \\
& +\left(-3 f_{1 y y}+2 f_{2 x y}-f_{3 x x}\right) V_{x}+\left(-f_{2 y y}+2 f_{3 x y}-3 f_{4 x x}\right) V_{y} .
\end{aligned}
$$

Дальнейшие нелинейные условия согласования для потеншиалов могут быть получены из системы (2.7)-(2.10). Чтобы записать их в компактном виде, введем функции

$$
\begin{aligned}
\phi_{1}= & \frac{V_{y}}{V_{x}}, \\
\phi_{2}= & -\hbar^{2} \frac{1}{4 V_{x}}\left(f_{1} V_{x x x}+f_{2} V_{x x y}+f_{3} V_{x y y}+f_{4} V_{y y y}+\right. \\
& \left.+8 A_{300}\left(x V_{y}-y V_{x}\right)+2\left(A_{210} V_{x}+A_{201} V_{y}\right)\right)
\end{aligned}
$$

и будем использовать обозначения $h_{1}, h_{2}$ и $h_{3}$ из формул $(2.8)-(2.10)$. Три алгебраичес- 
ки независимых нелинейных условия согласования теперь примут вид

$$
\begin{gathered}
-\phi_{2 x}+\left(\frac{\phi_{1}\left(h_{3} \phi_{1}+h_{2} \phi_{1}^{2}+\phi_{1} \phi_{2 y}+\phi_{2 x}+h_{1}\right)}{\phi_{1 x}+\phi_{1} \phi_{1 y}}\right)_{x}=h_{1}, \\
\left(\frac{\phi_{1}^{2} h_{2}+\phi_{1} \phi_{2 y}+\phi_{1} h_{3}+\phi_{2 x}+h_{1}}{\phi_{1 x}+\phi_{1} \phi_{1 y}}\right)_{y}=-h_{2}, \\
h_{1}\left(\phi_{1 x y}+\phi_{1 y}^{2}\right)+h_{2}\left(\phi_{1}^{2} \phi_{1 x y}-\phi_{1 x}^{2}-2 \phi_{1} \phi_{1 x} \phi_{1 y}\right)+ \\
+h_{3}\left(\phi_{1} \phi_{1 x y}-\phi_{1 x} \phi_{1 y}\right)-\left(h_{1 y}+\phi_{1} h_{2 x}\right)\left(\phi_{1 x}+\phi_{1} \phi_{1 y}\right)= \\
=-\phi_{2 x}\left(\phi_{1 x y}+\phi_{1 y}^{2}\right)+\phi_{2 y}\left(\phi_{1 x} \phi_{1 y}-\phi_{1 x y} \phi_{1}\right)+\phi_{2 x y}\left(\phi_{1 x}+\phi_{1} \phi_{1 y}\right) .
\end{gathered}
$$

Они представляют собой весьма сложные нелинейные уравнения пятого порядка (в классической механике - третьего порядка), и при обсуждении частных решений часто оказывается более удобным вернуться к исходным уравнениям (2.7)-(2.10), чтобы найти более простые условия согласования более низких порядков. Кроме того, при выводе общих условий согласования предполагалось

$$
\begin{array}{r}
\phi_{1 x}+\phi_{1} \phi_{1 y} \neq 0, \\
\phi_{1 x y} \phi_{1}-\phi_{1 x} \phi_{1 y} \neq 0,
\end{array}
$$

что не выполняется в случае исследуемых в данной работе потенциалов.

\section{3. ПОТЕНЦИАЛЫ, ДОПУСКАЮШИЕ СУШЕСТВОВАНИЕ ИНТЕГРАЛОВ ДВИЖЕНИЯ ПЕРВОГО И ТРЕТЬЕГО ПОРЯДКОВ}

Условия существования интеграла первого порядка в классической и квантовой механике совпадают и оказываются весьма ограничиваюшими. С точностью до сдвигов и поворотов гамильтонианами, допускающими сушествование интегралов первого порядка в двумерном евклидовом пространстве, оказываются только сферически-симметричные (радиальные) потенциалы $V=V(r)$ и трансляционно-инвариантные потенциалы $V=V(x)$. Очень немногие из них одновременно допускают сушествование нетривиального интеграла второго порядка. Из радиальных потенциалов это кулоновский потенциал $V=\alpha / r$ и потенциал гармонического осциллятора $V=\omega^{2} r^{2}$. Это следует из теоремы Бертрана, утверждаюшей, что лишь для этих двух потенциалов все ограниченные траектории замкнуты. Трансляционно-инвариантными потенциалами, имеющими интегралы второго порядка, оказываются лиш потенциалы $V=a x$ и $V=a / x^{2}$. Разумеется, поскольку эти четыре потенциала имеют интегралы движения низших порядков, у них также имеются зависимые интегралы движения третьего порядка, и они тем самым оказываются частными решениями как уравнений (2.3)-(2.6), так и уравнений (2.7)-(2.10).

В самом деле, при поиске систем с интегралами первого и третьего порядков в классической механике обнаруживаются только те же самые четыре потенциала, все интегралы движения которых следуют из интегралов более низких порядков. В радиальном случае это опять-таки следует из теоремы Бертрана. 
Ничего нового не удается найти в квантовой механике в случае потенщиала $V=V(r)$. Снова потенциалами, допускающими интегралы движения третьего порядка, оказываются только кулоновский потенциал и потенциал гармонического осциллятора. Это в точности совпадает с классическим случаем.

Но в случае $V=V(x)$ обнаруживается нечто новое. Конечно, потенциалы $V=a x$ и $V=a / x^{2}$ снова присутствуют в списке. Например, потенциал $V=a / x^{2}$ допускает сразу три интеграла движения третьего порядка

$$
\begin{aligned}
& X_{1}=\left\{L_{3}^{2}, p_{2}\right\}+2 a\left\{\frac{y^{2}}{x^{2}}, p_{2}\right\} \\
& X_{2}=\left\{L_{3}, p_{1} p_{2}\right\}-2 a\left\{\frac{y}{x^{2}}, p_{2}\right\} \\
& X_{3}=2 p_{1}^{2} p_{2}+2 a\left\{\frac{1}{x^{2}}, p_{2}\right\}
\end{aligned}
$$

которые следуют из интегралов движения более низких порядков и имеют классические аналоги. Новым оказывается то, что при специальных значениях параметра $a$, а именно при $V=\hbar^{2} / x^{2}$, у потенциала появляются четыре новых интеграла движения:

$$
\begin{aligned}
& X_{4}=2 L_{3}^{3}+\hbar^{2}\left\{\frac{3 y^{2}}{x}+2 x, p_{2}\right\}+\hbar^{2}\left\{-\frac{3 y^{3}}{x^{2}}-2 y, p_{1}\right\}, \\
& X_{5}=\left\{L_{3}^{2}, p_{1}\right\}-2 \hbar^{2}\left\{\frac{y}{x}, p_{2}\right\}+\frac{\hbar^{2}}{2}\left\{\frac{6 y^{2}}{x^{2}}+1, p_{1}\right\}, \\
& X_{6}=\left\{L_{3}, p_{1}^{2}\right\}+\hbar^{2}\left\{\frac{1}{x}, p_{2}\right\}-3 \hbar^{2}\left\{\frac{y}{x^{2}}, p_{1}\right\}, \\
& X_{7}=2 p_{1}^{3}+3 \hbar^{2}\left\{\frac{1}{x^{2}}, p_{1}\right\} .
\end{aligned}
$$

Они не являются независимыми один от другого и от предшествуюших интегралов, но, как утверждает Хиетаринта [2]-[4], [14], само существование подобных коммутирующих интегралов с нетривиальными алгебраическими соотношениями между ними оказывается полезным. Это свойство свидетельствует о том, что в такой ситуации надо рассматривать различные определения интегрируемости или суперинтегрируемости.

Если потенциал $V$ трансляционно-инвариантен, то можно найти новые суперинтегрируемые потенциалы, являющиеся решениями уравнения

$$
\hbar^{2} V_{x x x}=4(3 V+\alpha) V_{x},
$$

рассматриваемого в терминах эллиптической функции Вейерштрасса. Решения при этом выражаются через различные эллиптические функции Якоби, а именно

$$
\begin{aligned}
V_{i} & =(\hbar \omega)^{2} k \mathrm{sn}^{2}(\omega x, k), \\
V_{i i} & =\frac{(\hbar \omega)^{2}}{\operatorname{sn}^{2}(\omega x, k)} \\
V_{i i i} & =\frac{(\hbar \omega)^{2}}{2(\operatorname{cn}(\omega x, k)+1)},
\end{aligned}
$$


где $0 \leqslant k \leqslant 1, \omega \in \mathbb{R}$

Некоторые случаи можно представить в терминах элементарных функций. Например, имеется "солитонный" потенциал, представляющий собой специальный случай потенциала $V_{1}$ с $k=1$ :

$$
V_{i, a}=-\frac{(\hbar \omega)^{2}}{\operatorname{ch}^{2}(\omega x)} .
$$

Полагая $k=0$ или $k=1$ в $V_{2}$, можно получить соответственно периодический и непериодический сингулярные потенциалы:

$$
\begin{aligned}
V_{i i, a} & =\frac{(\hbar \omega)^{2}}{\sin ^{2}(\omega x)}, \\
V_{i i, b} & =\frac{(\hbar \omega)^{2}}{\operatorname{sh}^{2}(\omega x)} .
\end{aligned}
$$

Соответствующие интегралы имеют вид

$$
\begin{aligned}
& X_{1}=\left\{L_{3}, p_{1}^{2}\right\}-\left\{(3 V(x)+\alpha) y, p_{1}\right\}+\left\{\alpha x+2 x V(x)+\int V(x) d x, p_{2}\right\}, \\
& X_{2}=p_{1}^{3}+\frac{1}{2}\left\{3 V(x)+\alpha, p_{1}\right\} .
\end{aligned}
$$

На этом список потенциалов, допускающих существование интегралов первого и третьего порядка, исчерпан.

\section{4. ПОТЕНЦИАЛЫ, ДОПУСКАЮШИЕ РАЗДЕЛЕНИЕ ПЕРЕМЕННЫХ В ДЕКАРТОВЫХ КООР ДИНАТАХ}

Если положить $V=V_{1}(x)+V_{2}(y)$ в уравнениях (2.7)-(2.10), то получим

$$
\begin{gathered}
0=g_{1} V_{1 x}+g_{2} V_{2 y}-\frac{\hbar^{2}}{4}\left(f_{1} V_{1 x x x}+f_{4} V_{2 y y y}+\right. \\
\left.+8 A_{300}\left(x V_{2 y}-y V_{1 x}\right)+2\left(A_{210} V_{1 x}+A_{201} V_{2 y}\right)\right), \\
\left(g_{1}\right)_{x}=3 f_{1}(y) V_{1 x}+f_{2}(x, y) V_{2 y}, \\
\left(g_{2}\right)_{y}=f_{3}(x, y) V_{1 x}+3 f_{4}(x) V_{2 y}, \\
\left(g_{1}\right)_{y}+\left(g_{2}\right)_{x}=2\left(f_{2}(x, y) V_{1 x}+f_{3}(x, y) V_{2 y}\right) .
\end{gathered}
$$

Уравнения (4.2) и (4.3) явно интегрируются; тем самым, в декартовом случае остается решить еше два уравнения.

Из линейного условия согласования следует, что

$$
\begin{gathered}
\left(A_{210} x^{2}+A_{111} x+A_{012}\right) V_{1}^{(3)}(x)+4\left(2 A_{210} x+A_{111}\right) V_{1}^{\prime \prime}(x)+12 A_{210} V_{1}^{\prime}(x)=a x+b, \\
\left(A_{201} y^{2}-A_{111} y+A_{021}\right) V_{2}^{(3)}(y)+4\left(2 A_{201} y-A_{111}\right) V_{2}^{\prime \prime}(y)+12 A_{201} V_{2}^{\prime}(y)=c y+d .
\end{gathered}
$$


Среди решений системы уравнений (4.1)-(4.4) находятся все квадратично-суперинтегрируемые системы, но помимо них возникает много новых потенциалов (преимущественно в случае квантовой механики, но некоторые и в классическом случае). Во-первых, отметим анизотропный гармонический осциллятор

$$
\begin{aligned}
V & =a\left(9 x^{2}+y^{2}\right), \\
X_{1} & =\left\{L, p_{2}^{2}\right\}+\frac{2}{3} a\left\{y^{3}, p_{1}\right\}-6 a\left\{x y^{2}, p_{2}\right\},
\end{aligned}
$$

которьй, как и ожидалось, имеет интеграл движения третьего порядка. Тем не менее заметим, что этот потенциал, суперинтегрируемость которого уже давно была известна [16], не возникал в списке классических потенциалов с интегралами третьего порядка, выписанном Драчом в работе [11].

В классическом случае возникают и более экзотические потенциалы, например

$$
\begin{aligned}
V & =a y+b \sqrt{x} \\
X_{1} & =p_{1}^{3}+3 b \sqrt{x} p_{1}-\frac{3 b^{2}}{2 a} p_{2},
\end{aligned}
$$

найденный также в работе [13]. Отметим, что интеграл движения $X_{1}$ не зависит от интегралов второго порядка

$$
\begin{aligned}
& H_{1}=\frac{p_{1}^{2}}{2}+b \sqrt{x}, \\
& H_{2}=\frac{p_{2}^{2}}{2}+a y,
\end{aligned}
$$

что следует из присутствия в нем члена, зависящего от $p_{2}$, и того, что $\partial_{y} X_{1}=0$.

В квантовой механике ситуация опять оказывается более сложной. Во многих случаях потенциалы со специальными величинами параметров порождают новые интегралы, как происходит при $V=a / x^{2}$. Возникает также много потенциалов, которые можно записать в виде сумм суперинтегрируемых потенциалов, например,

$$
\begin{aligned}
V= & \hbar^{2}\left(\frac{2}{\alpha^{4}}\left(x^{2}+y^{2}\right)+\frac{1}{y^{2}}+\frac{1}{x^{2}}+\frac{1}{(x-\alpha)^{2}}-\frac{2 x}{\alpha^{3}}\right), \\
X_{1}= & 2 L^{3}+2 \frac{\alpha^{3}}{4} p_{2}^{3}-\frac{3 \alpha}{2}\left\{L^{2}, p_{2}\right\}+ \\
& +\hbar^{2}\left\{-\frac{3 x^{2}}{y}-2 y-3 \frac{y^{3}}{x^{2}}+3 y^{3}\left(-\frac{1}{(x-\alpha)^{2}}+\frac{1}{\alpha^{2}}\right)+\frac{3 x \alpha}{y}, p_{1}\right\}+ \\
& +\frac{\hbar^{2}}{4}\left\{8 x-12 x y^{2}\left(-\frac{1}{x^{3}}-\frac{1}{(x-\alpha)^{3}}+\frac{2 x}{\alpha^{4}}-\frac{1}{\alpha^{3}}\right)(x-\alpha)-\right. \\
& \left.-3 \alpha+3 \frac{\left(4 x^{3}-6 x^{2} \alpha+\alpha^{3}\right)\left(2 y^{4}+\alpha^{4}\right)}{\alpha^{4} y^{2}}, p_{2}\right\} .
\end{aligned}
$$


В случае, когда линейное условие согласования удовлетворяется тривиально, имеют место нелинейные уравнения, из которых можно получить более интересные потенциалы. Например, можно найти, что потенциал

$$
V=a y+\hbar^{2} P_{1}(x, \lambda)
$$

допускает интеграл движения

$$
X=2 p_{1}^{3}+3 \hbar^{2}\left\{P_{1}(x, \lambda), p_{1}\right\}+\hbar^{4}\left\{\frac{\lambda}{4 a}, p_{2}\right\} .
$$

Здесь $P_{1}$ есть первый трансцендент Пенлеве, задаваемый уравнением

$$
P_{1}^{\prime \prime}(x, \lambda)=6 P_{1}^{2}(x, \lambda)+\lambda x .
$$

Заметим, что потенциал (4.7) получается как специальный случай потенциала (4.10) после замены $\lambda \rightarrow \lambda / \hbar^{4}$ и последуюшего взятия предела $\hbar \rightarrow 0$.

\section{5. ЗАКЛЮЧЕНИЕ}

Большинство из найденных суперинтегрируемых потенциалов может быть записано в виде сумм четырех "основных" суперинтегрируемых потенциалов (гармонического осциллятора, кулоновского потенциала, потенциалов $V=a x$ и $\left.V=a / x^{2}\right)$. Остальные потенциалы либо выражаются через элементарные функции, либо представляют собой решения уравнений, обладаюших свойством Пенлеве. За прошедшие годы нелинейные уравнения, обладающие свойством Пенлеве, были объектом тщательного исследования. То, что такие потенциалы появляются как решения уравнений, обладаюших свойством Пенлеве, само по себе интересно и, возможно, не случайно. Также интересно, что в этом новом подходе появились трансцендентные функции Пенлеве.

В данной работе вновь было обнаружено, что число интегралов движения может меняться скачками при вариациях параметров как в квантовой, так и в классической механике. Давно уже было известно, что некоторые максимально суперинтегрируемые потенциалы допускают больше интегралов движения, чем другие; прекрасным (хотя и тривиальным) примером таких потенциалов служит потенциал свободного движения. В случае одномерного движения, когда все потенциалы суперинтегрируемы по определению, было показано, что некоторые из них допускают сушествование интегралов, нетривиально связанных с гамильтонианом [2]. Это те же самые потенциалы, которые были найдены в двумерном случае, рассмотренном в данной работе, когда искались потенциалы с зависимостью $V=V(x)$. Поэтому оказывается, что только одномерные потенциалы, допускающие существование нетривиально зависимых интегралов движения третьего порядка, одновременно допускают существование независимых интегралов движения в двумерном плоском пространстве, т.е. сушествование интегралов с нетривиальными алгебраическими соотношениями связано с существованием подлинно независимых интегралов движения в пространстве высшей размерности. Это дает еше одно указание на то, что обычное определение интегрируемости может быть недостаточным для описания всех различий между потенциалами, следующих из подсчета числа интегралов движения. 
Благодарности. Автор глубоко признателен П. Винтерницу за весьма полезные комментарии и предложения. Работа была поддержана стипендией NSERC ES-A.

\section{Список литературы}

[1] В. И. Арнольд. Математические методы классической механики. М.: Наука, 1979; Г. Голдстейн. Классическая механика. М.: Наука, 1974.

[2] J. Hietarinta. Phys. Lett. A. 1998. V. 246. P. 97-104.

[3] J. Hietarinta. J. Math. Phys. 1984. V. 25. P. 1833-1840.

[4] J. Hietarinta. J. Phys. A. 1989. V. 22. P. L143-L147.

[5] M. B. Sheftel, P. Tempesta, P. Winternitz. J. Math. Phys. 2001. V. 42. P. 659-673.

[6] M. A. Rodriguez, P. Winternitz. J. Math. Phys. 2002. V. 43. P. 1309-1322.

[7] N. W. Evans. Phys. Rev. A. 1990. V. 41. P. 5666-5676; J. Math. Phys. 1991. V. 32. P. 3369-3375; Phys. Lett. A. 1990. V. 147. P. 483-486; A. Makarov, J. Smorodinsky, Kh. Valiev, P. Winternitz. Nuovo Cimento A. 1967. V. 52. P. 1061-1084; П. Винтернич, Я. Смородинский, М. Ульрих, И. Фриз. ЯФ. 1966. Т. 4. С. 625-635.

[8] E. G. Kalnins, W. Miller Jr., G. S. Pogosyan. J. Phys. A. 2001. V. 34. P. 4705-4720; M. F. Rañada, M. Santander. J. Math. Phys. 1999. V. 40. P. 5026-5057.

[9] E. G. Kalnins, J. M. Kress, P. Winternitz. J. Math. Phys. 2002. V. 43. P. 970-983.

[10] P. Tempesta, A. V. Turbiner, P. Winternitz. J. Math. Phys. 2001. V. 42. P. 4248-4257.

[11] J. Drach. C. R. Acad. Sci. 1935. V. 200. P. 22.

[12] C. R. Holt. J. Math. Phys. 1982. V. 23. P. 1037-1046; A. S. Fokas, P. A. Lagerstrom. J. Math. Anal. Appl. 1980. V. 74. P. 325-341; P. 342-358; C. Gonera, P. Kosinsky, P. Maslanka. Phys. Lett. A. 2001. V. 289. P. 66-68; M. Karlovini, K. Rosquist. J. Math. Phys. 2000. V. 41. P. 370-384.

[13] A. V. Tsiganov. J. Phys. A. 2000. V. 33. P. 7407-7422.

[14] J. Hietarinta, B. Grammaticos. J. Phys. A. 1989. V. 22. P. 1315-1322.

[15] S. Gravel, P. Winternitz. Superintegrable systems with third-order integrals in classical and quantum mechanics. J. Math. Phys. (to appear).

[16] J. Jauch, E. Hill. Phys. Rev. 1940. V. 51. P. 641-645. 